\title{
EMISIÓN DIFUSA DE DIÓXIDO DE CARBONO Y VAPOR DE MERCURIO EN EL VOLCÁN MIRAVALLES, COSTA RICA
}

\author{
Gladys V. Melián ${ }^{1 *}$, Nemesio M. Pérez ${ }^{1}$, Pedro A. Hernández ${ }^{1}$, \\ José M. L. Salazar ${ }^{1}$, Antonio Yock ${ }^{2}$, Eddy Sánchez ${ }^{2}$, \\ Guillermo E. Alvarado ${ }^{2}$, Hirochika Sumino ${ }^{3}$ \& Kenji Notsu ${ }^{3}$ \\ ${ }^{1}$ División de Medio Ambiente, Instituto Tecnológico y de Energías Renovables \\ (ITER), 38611 Granadilla, S/C de Tenerife, España \\ 2 Instituto Costarricense de Electricidad, Apdo. 10032-1000, San José, Costa Rica \\ ${ }^{3}$ Laboratory for Earthquake Chemistry, University of Tokyo, \\ Bunkyo-ku, Tokio 113, Japón \\ * Autora para contacto: gladys@iter.rcanaria.es
}

(Recibido 12/06/03; aceptado 03/09/03)

\begin{abstract}
The purpose of this study is to evaluate the spatial distribution of diffuse $\mathrm{CO}_{2}$ and elemental $\mathrm{Hg}$ degassing at Miravalles volcano as well as to estimate the total output $\mathrm{CO}_{2}$ efflux. Relatively high soil $\mathrm{CO}_{2}$ efflux measurements and soil vapor $\mathrm{Hg}^{0}$ concentrations were observed at Miravalles, reaching values up to $24 \mathrm{~kg} \mathrm{~m}^{-2}$ $\mathrm{d}^{-1}$, and $25 \mathrm{ppbV}$, respectively. Anomalous soil $\mathrm{CO}_{2}$ efflux, soil vapor $\mathrm{Hg}^{0}$ values and relatively heavy carbon isotopic signatures of carbon for the soil gas $\mathrm{CO}_{2}$ were spatially well correlated with major volcano-structural characteristics of Miravalles. Diffuse $\mathrm{CO}_{2}$ degassing rate of the study area yields $12700 \mathrm{t} \mathrm{d}^{-1}$. These results are useful to improve the geochemical monitoring program for the volcanic surveillance of Miravalles.
\end{abstract}

Keywords: Miravalles volcano, diffuse emission, carbon dioxide, mercury vapor, volcanic surveillance, carbon isotopes, volcanic structures.

RESUMEN: En este trabajo se presenta un estudio sobre desgasificación difusa de $\mathrm{CO}_{2}$ y vapor de mercurio $\left(\mathrm{Hg}^{0}\right)$ en el volcán Miravalles, así como una estimación conservativa de la tasa de emisión de $\mathrm{CO}_{2}$ a la atmósfera. Valores relativamente altos de flujo difuso de $\mathrm{CO}_{2}$ y concentración de vapor de $\mathrm{Hg}^{0}$ se han observado en el volcán Miravalles, llegándose a alcanzar valores del orden de los $24 \mathrm{~kg} \mathrm{~m}^{-2} \mathrm{~d}^{-1}$ y $25 \mathrm{ppbV}$, respectivamente. Los resultados reflejan la existencia de una buena correlación espacial entre las anomalías de flujo difuso de $\mathrm{CO}_{2}$, concentración de vapor de $\mathrm{Hg}^{0}$ y las relaciones isotópicas de carbono en el $\mathrm{CO}_{2}$ de la atmósfera del suelo, con las principales características volcano-estructurales de Miravalles. La cantidad de $\mathrm{CO}_{2}$ emitida de forma difusa a la atmósfera por el área de estudio es de $12700 \mathrm{t} \mathrm{d}^{-1}$. Estos resultados son muy útiles para mejorar el programa geoquímico destinado a la vigilancia volcánica de Miravalles.

Palabras clave: Volcán Miravalles, emisión difusa, dióxido de carbono, vapor de mercurio, vigilancia volcánica, isótopos de carbono, estructuras volcánicas. 


\section{INTRODUCCIÓN}

Durante las últimas décadas se han realizado numerosos estudios de volátiles en áreas volcánicas para evaluar los procesos de desgasificación magmática y sus variaciones temporales, demostrando su estrecha relación con el grado de actividad del sistema volcánico (Hirabayashi et al., 1982; Giggenbach, 1987, 1996; Martini et al., 1991). Dentro de esta temática, la medida y evaluación de la desgasificación difusa de $\mathrm{CO}_{2}$ ha cobrado un especial interés durante los últimos años (Baubron et al., 1990; Pérez et al., 1996; Chiodini et al., 1996; Hernández et al., 2001a, 2001b; Rogie et al., 2001; Salazar et al., 2001, 2002). Este tipo de estudios se han enfocado en el $\mathrm{CO}_{2}$ por ser un excelente trazador para evaluar los procesos de desgasificación magmática, debido a que es la segunda especie mayoritaria en los gases volcánicos después del vapor de agua y a su baja solubilidad en fundidos silicatados a presiones bajas o moderadas, lo que facilita su escape a la atmósfera (Gerlach \& Graeber, 1985; Giggenbach, 1996). Las anomalías de gases y volátiles en el ambiente superficial del suelo están controladas principalmente por las condiciones termodinámicas del sistema volcánico hidrotermal, y por las características volcanoestructurales, ya que los volátiles pueden escapar a la atmósfera con mayor facilidad a través de fallas o fracturas. La evolución temporal y espacial de estas anomalías pueden estar relacionadas con cambios en la actividad del sistema volcánico; por lo tanto, el monitoreo de la distribución espacial de estas emanaciones, puede ser de gran ayuda para identificar lugares en los cuales la probabilidad de que ocurra una erupción sea mayor (Hernández et al., 2001b).

El volcán Miravalles está localizado en la Cordillera de Guanacaste, situada al noreste del país y aproximadamente a $150 \mathrm{~km}$ al norte de San José (Fig 1). Es un estratovolcán cuaternario muy complejo $\left(160 \mathrm{~km}^{2}\right)$, en cuya cima se encuentran cuatro cráteres alineados en dirección NE-SO. Con una altura de 2028 m s.n.m., es el más alto de la Cordillera de Guanacaste. El ciclo eruptivo más reciente tuvo lugar hace 9000 años con excepción de una pequeña explosión freatomagmática en el año

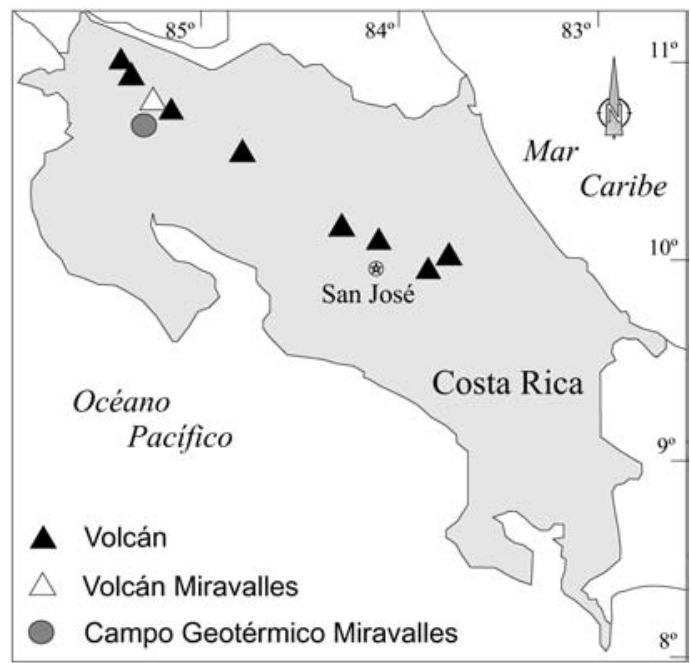

Fig. 1: Localización del volcán Miravalles en Costa Rica.

1946, localizada en el flanco sureste del volcán, cerca de la zona de Las Hornillas. Es esta área donde se localiza actualmente la actividad volcánica, caracterizada por la existencia de fumarolas, fuentes termales y hervideros de lodo con temperaturas que alcanzan los $94^{\circ} \mathrm{C}$ (Alvarado, 2000). Al sureste del volcán Miravalles se puede observar un conjunto de fallas secundarias y el graben de La Fortuna, donde está emplazado el Proyecto Geotérmico Miravalles, que aprovecha los recursos geotérmicos del subsuelo para la generación de energía eléctrica.

El objetivo de este trabajo es estudiar los niveles de desgasificación difusa de $\mathrm{CO}_{2}$ y vapor de $\mathrm{Hg}^{0}$ en el volcán Miravalles, con el fin de identificar las principales zonas de desgasificación y estimar la cantidad de $\mathrm{CO}_{2}$ emitida de forma difusa a la atmósfera por este sistema volcánico. Esta información es de especial importancia para el desarrollo del programa de vigilancia volcánica en el Miravalles.

\section{METODOLOGÍA}

En febrero del 2000 se realizó una campaña en el volcán Miravalles consistente en la toma de muestras de gas del suelo y la realización de medidas in-situ de vapor de $\mathrm{Hg}^{0}$ y flujo difuso de 
$\mathrm{CO}_{2}$ en el ambiente superficial. El número total de puntos de observación en esta campaña fue de 244, distribuidos en un área de $65 \mathrm{~km}^{2}$. La selección de los puntos de observación se realizó siguiendo las características geológicas y estructurales del sistema volcánico, así como criterios de accesibilidad del área, presentándose muy difícil trabajar en cotas altas (> $1400 \mathrm{~m}$ ) y en el flanco noreste del volcán, debido a la vegetación existente en la zona y la poca accesibilidad a ella.

Las medidas de flujo difuso de $\mathrm{CO}_{2}$ se realizaron in-situ, siguiendo el método de la "cámara de acumulación" (Chiodini et al., 1996). Para ello, se utilizó un sensor portátil de infrarrojo no dispersivo (NDIR) modelo Riken 411A. El rango de reproducibilidad de nuestras medidas se encuentra entre 10 y $35000 \mathrm{~g} \mathrm{~m}^{-2} \mathrm{~d}^{-1}$, asumiendo un error de $\pm 10 \%$, valor basado en la variabilidad de las réplicas realizadas en el laboratorio. Los valores de flujo de $\mathrm{CO}_{2}$ son determinados por el incremento de la concentración de $\mathrm{CO}_{2}$ medida dentro de la cámara de acumulación respecto al tiempo. Los valores de flujo difuso de $\mathrm{CO}_{2}$ se corrigieron con los datos de presión y temperatura atmosférica tomados en cada punto de muestreo para convertir concentraciones volumétricas en concentraciones másicas.

Las muestras de gas del suelo se recogieron a una profundidad de $40 \mathrm{~cm}$ usando una sonda metálica insertada previamente. El gas se extrae con una jeringuilla hipodérmica de $30 \mathrm{~cm}^{3}$, transfiriendo posteriormente una alícuota a un tubo de vidrio sellado de $10 \mathrm{~cm}^{3}$ (Hinkle \& Kilburn, 1979), utilizando el método de desplazamiento de agua para transferir la alícuota de gas al tubo de vidrio. El análisis de las muestras de gas de suelo se realizó diariamente en el laboratorio de campo instalado en el Campo Geotérmico Miravalles mediante un microcromatógrafo VARIAN modelo $\mathrm{CP}$ 2002P. Las medidas de concentración de $\mathrm{Hg}^{0}$ también se realizaron $i n$-situ, mediante un sensor portátil Jerome 431-X, tomando una alícuota de 60 $\mathrm{cm}^{3}$ de gas del suelo. Las relaciones isotópicas ${ }^{13} \mathrm{C} /{ }^{12} \mathrm{C}$ en el $\mathrm{CO}_{2}$ de la atmósfera del suelo fueron determinadas por un espectrómetro de masas Finnigan modelo MAT delta-S. La relación ${ }^{13} \mathrm{C} /{ }^{12} \mathrm{C}$ se expresa como $\delta^{13} \mathrm{C}(\%)$ con respecto a un estándar internacional de carbonato V-PBD. El error analítico asociado con la medida de las relaciones isotópicas ${ }^{13} \mathrm{C} /{ }^{12} \mathrm{C}$ fue de $\pm 0,1 \%$.

En la zona de Las Hornillas, se recogió una muestra de gas fumarólico para determinar el contenido y la firma isotópica del $\mathrm{CO}_{2}$ y $\mathrm{He}$. La medidas de los isótopos de He se realizaron mediante el uso de un espectrómetro de masas de alta precisión (VB5400, VG Isotopes). El He atmosférico es utilizado como estándar, siendo el error en las relaciones $\mathrm{R} / \mathrm{Ra}$ de un $\pm 1 \%$ (donde $\mathrm{Ra}$ es la relación ${ }^{3} \mathrm{He} /{ }^{4} \mathrm{He}$ para el aire atmosférico, con un valor de $1,4 \times 10^{-6}$ ). Las relaciones ${ }^{4} \mathrm{He} /{ }^{20} \mathrm{Ne}$ son medidas en un espectrómetro de masas cuadrupolar (QMG 112, Blazer) utilizando aire como estándar. Los errores en la medida de las relaciones ${ }^{4} \mathrm{He} /{ }^{20} \mathrm{Ne}$ son estimados en \pm $10 \%$. Los análisis de isótopos de carbono y de helio, así como la relación ${ }^{4} \mathrm{He} /{ }^{20} \mathrm{Ne}$ se realizaron en el Laboratorio de Química de Terremotos de la Universidad de Tokio.

\section{RESULTADOS Y DISCUSIÓN}

En el Cuadro 1 se presenta una estadística descriptiva simple de los resultados de los análisis de las muestras de gas recogidos en el volcán Miravalles. Los valores de flujo difuso de $\mathrm{CO}_{2}$

Cuadro 1

Caracterización química, isotópica y físico-química

de las emanaciones difusas de gases en el volcán Miravalles (febrero 2000)

\begin{tabular}{lcccccccccc}
\hline & $\begin{array}{c}\mathrm{He} \\
(\mathrm{ppmV})\end{array}$ & $\begin{array}{c}\mathrm{H}_{2} \\
(\mathrm{ppmV})\end{array}$ & $\begin{array}{c}\mathrm{CH}_{4} \\
(\mathrm{ppmV})\end{array}$ & $\begin{array}{c}\mathrm{O}^{2} \\
(\%)\end{array}$ & $\begin{array}{c}\mathrm{N}_{2} \\
(\%)\end{array}$ & $\begin{array}{c}\mathrm{CO}_{2} \\
(\%)\end{array}$ & $\begin{array}{c}\mathrm{Hg}^{0} \\
(\mathrm{ppbV})\end{array}$ & $\begin{array}{c}\delta^{13} \mathrm{C}_{-} \mathrm{CO}_{2} \\
(\%)\end{array}$ & $\begin{array}{c}\Phi \mathrm{CO}_{2} \\
\left(\mathrm{~g} \mathrm{~m}^{-2} \mathrm{~d}^{-1}\right)\end{array}$ \\
\hline Mínimo & 0,784 & 0,051 & 0,52 & 15,8 & 77,9 & 0,03 & 0 & $-35,1$ & 0,4 \\
Máximo & 8,294 & 0,60 & 2,47 & 21,5 & 81,8 & 57,6 & 25 & $-1,3$ & 24153 \\
Mediana & 5,377 & 0,389 & 1,15 & 20,2 & 80,7 & 0,60 & 1,01 & $-23,1$ & 255 \\
\hline
\end{tabular}


presentaron un rango de medidas comprendido entre valores por debajo del límite de detección (< $\left.0,5 \mathrm{~g} \mathrm{~m}^{-2} \mathrm{~d}^{-1}\right)$ hasta los $24153 \mathrm{~g} \mathrm{~m}^{-2} \mathrm{~d}^{-1}$, con una mediana de $255 \mathrm{~g} \mathrm{~m}^{-2} \mathrm{~d}^{-1}$. Los valores de concentración de $\mathrm{CO}_{2}$ en la atmósfera del suelo presentaron también un amplio rango entre un 0,03 y un $57,6 \%$, al igual que la firma isotópica de $\mathrm{CO}_{2}$ con valores de $\delta^{13} \mathrm{C}\left(\mathrm{CO}_{2}\right)$ desde $-35,1 \mathrm{a}-1,3 \%$ o. En el caso de las concentraciones de vapor de $\mathrm{Hg}^{0}$ en la atmósfera del suelo, el rango de valores observados se encuentra entre 0 y $24,9 \mathrm{ppbV}$.

Con el objeto de identificar diferentes poblaciones geoquímicas se realizó un tratamiento estadístico-gráfico (Sinclair, 1974) de los datos de flujo difuso de $\mathrm{CO}_{2}$, observándose dos poblaciones: fondo y anómala. El valor medio obtenido para la población geoquímica de fondo fue de $5 \mathrm{~g} \mathrm{~m}^{-2} \mathrm{~d}^{-1}$ y representa el $96 \%$ del total de los datos. El valor medio de la población de fondo fue similar al observado para otros sistemas volcánicos, y corresponde principalmente a la actividad biogénica presente en el área de estudio (Hernández et al., 2001a). El valor medio para la población anómala fue de $1158 \mathrm{~g} \mathrm{~m}^{-2} \mathrm{~d}^{-1} \mathrm{y}$ representa un $4 \%$ del total de los datos. La presencia de diferentes poblaciones geoquímicas sugiere la existencia de perturbaciones de origen profundo en el ambiente superficial del sistema volcánicohidrotermal del volcán Miravalles.

Los resultados obtenidos para la concentración y flujo difuso de $\mathrm{CO}_{2}$, así como para la concentración de $\mathrm{Hg}^{0}$, fueron utilizados para la construcción de mapas de contorno mediante la técnica de interpolación Krigging, usando un variograma experimental (Journel, 1988; Cressie, 1990). La distribución espacial del flujo difuso de $\mathrm{CO}_{2}$ en el volcán Miravalles refleja que las principales anomalías de flujo difuso de $\mathrm{CO}_{2}$ se localizaron al SE y E del volcán, donde se observaron valores superiores a 10 y $5 \mathrm{~kg} \mathrm{~m}^{-2} \mathrm{~d}^{-1}$, respectivamente (Fig. 2). En el flanco SO se pudo observar una pequeña anomalía de flujo difuso de $\mathrm{CO}_{2}$ que alcanzó valores de $750 \mathrm{~g} \mathrm{~m}^{-2} \mathrm{~d}^{-1}$. Esta última anomalía coincide en el espacio con la existencia de un conjunto de fallas secundarias y el Graben de La Fortuna.

La distribución espacial de la concentración de $\mathrm{CO}_{2}$ en la atmósfera del suelo en el volcán

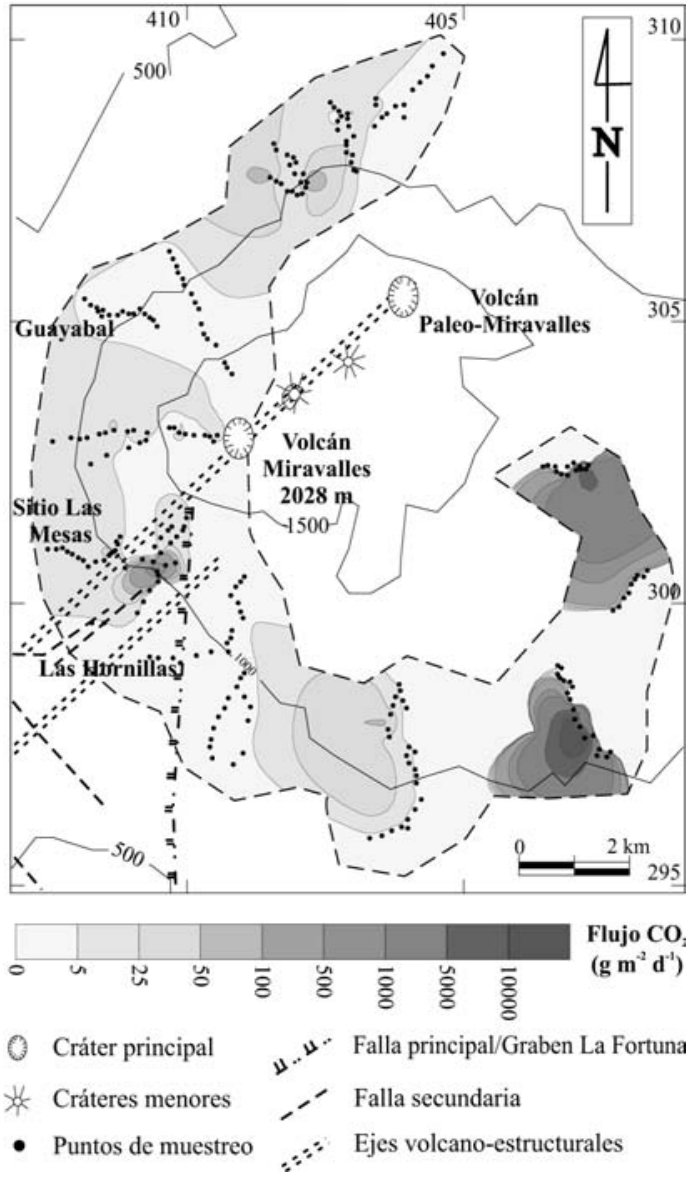

Fig. 2: Distribución espacial de los niveles de flujo difuso de dióxido de carbono en el volcán Miravalles, Costa Rica (febrero 2000).

Miravalles se observa en la figura 3. Los niveles de concentración de $\mathrm{CO}_{2}$ en el suelo fueron relativamente altos y presentaron un amplio rango de valores entre un 0,03 y un $57,6 \%$, con un valor medio de $0,6 \%$. La principal anomalía de concentración de $\mathrm{CO}_{2}$ en el suelo de Miravalles se detectó en el flanco SO, con concentraciones superiores a un $20 \%$, en la misma zona donde se detectaron valores anómalos de flujo difuso de $\mathrm{CO}_{2}$ que alcanzaron los $750 \mathrm{~g} \mathrm{~m}^{-2} \mathrm{~d}^{-1}$. Esta anomalía de desgasificación difusa de $\mathrm{CO}_{2}$ está perfectamente alineada con los ejes volcano-estructurales.

La distribución espacial de la firma isotópica de dióxido de carbono $\left(\delta^{13} \mathrm{C}-\mathrm{CO}_{2}\right)$ en la atmósfera del suelo (Fig. 4) refleja que las principales 

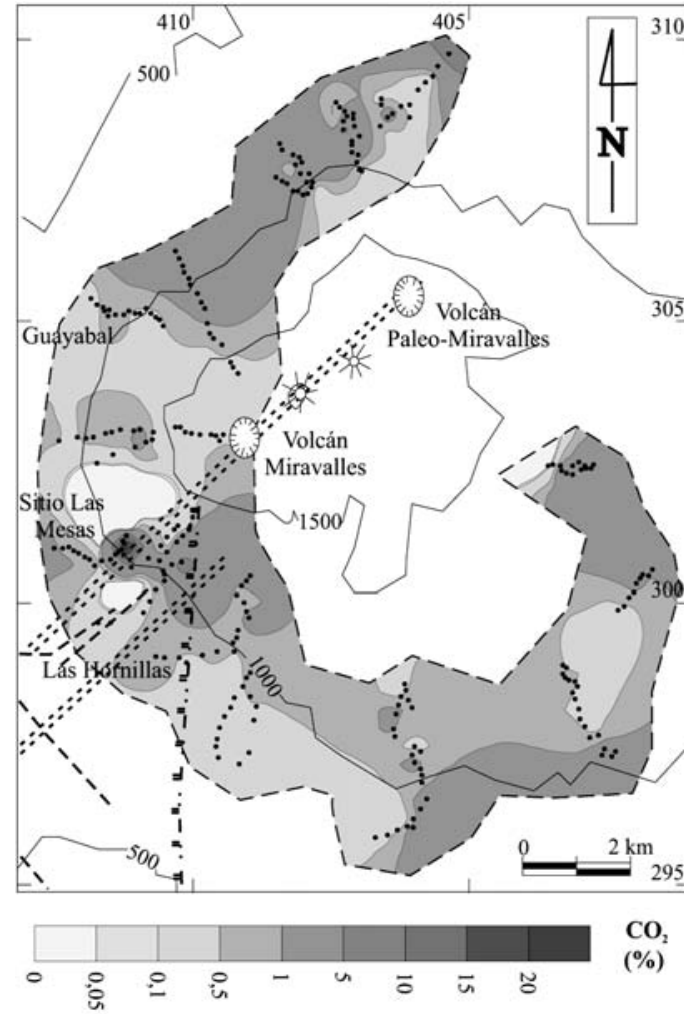

$\begin{array}{lll}\text { Cráter principal } & \text { Falla principal/Graben La Fortuna } \\ \text { * Cráteres menores } & \text { - Falla secundaria } \\ \text { - Puntos de muestreo } & \because \text { Ejes volcano-estructurales }\end{array}$

Fig. 3: Distribución espacial de los niveles de concentración de dióxido de carbono en el volcán Miravalles, Costa Rica (febrero 2000).

anomalías con valores superiores a $-4 \%$, se observan también en el flanco SO del volcán, alineadas con el Graben de La Fortuna. Estas anomalías están bien correlacionadas con anomalías de flujo difuso y concentración de $\mathrm{CO}_{2}$. La existencia de fallas en el flanco SO del volcán Miravalles, unido a la presencia de anomalías de gases y volátiles, sugiere la existencia de un área de mayor permeabilidad que permite el ascenso de gases de origen profundo a la superficie.

En la figura 5 se presenta la distribución espacial de los niveles de concentración de vapor de $\mathrm{Hg}^{0}$ para el volcán Miravalles. En los flancos $\mathrm{N}$ y $\mathrm{O}$ se detectaron las principales anomalías de $\mathrm{Hg}^{0}$, donde se llegaron a superar los $10 \mathrm{ppbV}$ de vapor de $\mathrm{Hg}^{0}$. Concentraciones superiores a $\operatorname{los} 8$

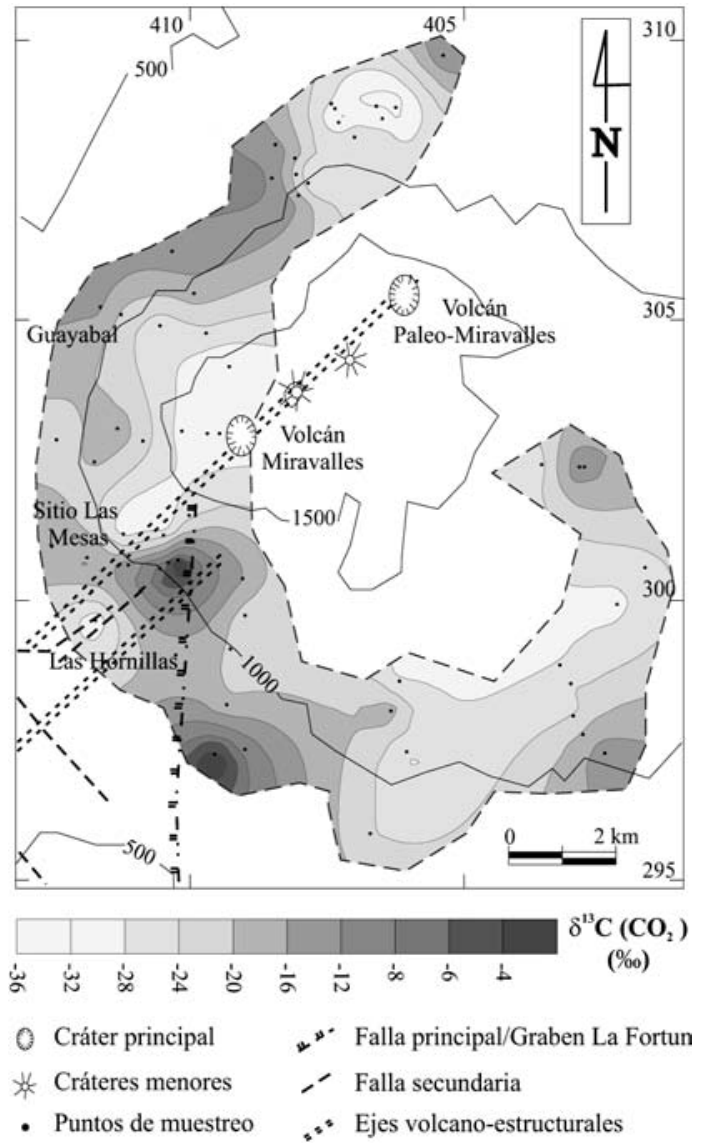

Fig. 4: Distribución espacial de los niveles de $\delta^{13} \mathrm{C}\left(\mathrm{CO}_{2}\right)$ en el gas del suelo del volcán Miravalles, Costa Rica (febrero 2000).

ppbV de vapor de $\mathrm{Hg}^{0}$ se localizaron en el flanco $\mathrm{S}$ y SO del volcán. Las concentraciones máximas de $\mathrm{Hg}^{0}$ medidas en el volcán Miravalles (25 ppbV) fueron muy inferiores a las observadas en el volcán Rincón de la Vieja, donde se encontraron máximos que alcanzaron los $180 \mathrm{ppmV}$ de vapor de $\mathrm{Hg}^{0}$ (Lescinsky et al., 1987). El estudio en el Rincón de la Vieja se localizó en la zona de Las Pailas, en el sector SO del volcán, donde existen numerosas fuentes termales. La diferencia existente entre los valores obtenidos en Miravalles y los de Rincón de la Vieja pueden explicarse por el diferente procedimiento analítico utilizado para el análisis del vapor de $\mathrm{Hg}^{0}$ contenido en el suelo. Lescinsky et al. (1987) recogieron muestras de suelo a unos 40 centímetros de profundidad que 


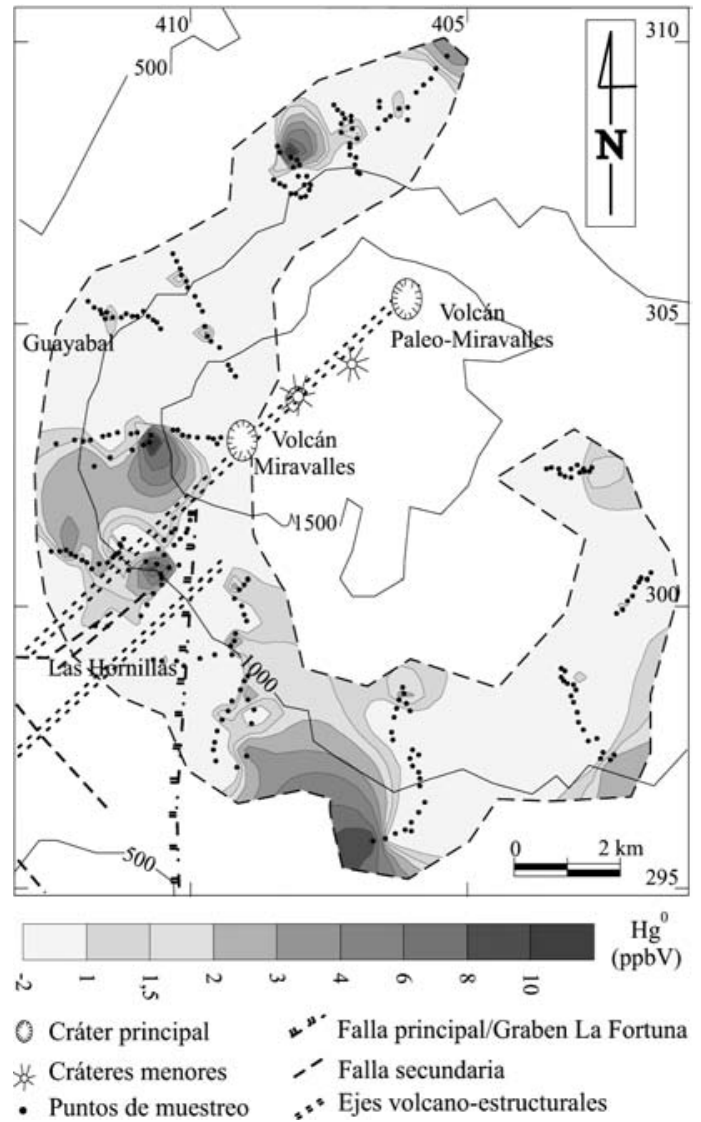

Fig. 5: Distribución espacial de los niveles de concentración de vapor de mercurio en el volcán Miravalles, Costa Rica (febrero 2000).

posteriormente fueron secadas al aire, tamizadas y calentadas a una temperatura de $800^{\circ} \mathrm{C}$ con la finalidad de liberar y medir el contenido de $\mathrm{Hg}^{0}$ en la muestra de suelo mediante un sensor tipo JEROME. Al calentar la muestra de suelo, se favorece la liberación del $\mathrm{Hg}^{0}$ retenido en la fracción orgánico-arcillosa del suelo, por lo que es de esperar que los valores obtenidos en Rincón de la Vieja sean muy superiores a los observados en Miravalles.

Paralelamente al estudio de emisiones difusas en el volcán Miravalles, se procedió a la toma de una muestra de gas fumarólico en Las Hornillas con la finalidad de caracterizar el origen de estas descargas volcánico-hidrotermales. La firma isotópica del $\mathrm{CO}_{2}$ y He que emiten las fumarolas reflejan valores de $\delta^{13} \mathrm{C}\left(\mathrm{CO}_{2}\right)=-1,8 \%$ y
${ }^{3} \mathrm{He} /{ }^{4} \mathrm{He}=5,00 \pm 0,13 \mathrm{Ra}$, mientras que la relación molar $\mathrm{CO}_{2} /{ }^{3} \mathrm{He}$ fue de $1,10 \times 10^{10}$. Aplicando un simple balance de masas para definir el origen del He (Sano, et al., 1985; Sano \& Marty, 1995), se estima que un $58 \%$ del He es de origen magmático mientras que un $4 \%$ es de origen radiogénico y un $38 \%$ de origen atmosférico. Estos resultados reflejan una importante contaminación atmosférica que explicaría la relativamente baja relación isotópica de $\mathrm{He}$ observada para el Miravalles en la muestra de gas recogida en Las Hornillas. La relación ${ }^{3} \mathrm{He} /{ }^{4} \mathrm{He}$ observada de 5,00 \pm $0,13 \mathrm{Ra}$ es inferior a las obtenidas por Snyder et al. (2001) para Miravalles, cuyos valores se encuentran entre 5,6 y 6,8 Ra para las emanaciones volcánicas-hidrotermales de este sistema volcánico. Con la finalidad de evaluar el origen del $\mathrm{CO}_{2}$ en el gas fumarólico de Las Hornillas combinamos la sistemática de isótopos de $\mathrm{CO}_{2}$ y $\mathrm{He}$. Un simple balance de masas (Sano, et al., 1985; Sano \& Marty, 1995) refleja que el $\mathrm{CO}_{2}$ de Las Hornillas se deriva en un $83 \%$ de carbonatos marinos, mientras que un 14 y $3 \%$ se deriva del manto y de sedimentos orgánicos, respectivamente. Estos resultados indican que una importante fracción del $\mathrm{CO}_{2}$ emitido por el volcán Miravalles es de origen profundo, reflejándose que una gran parte de este $\mathrm{CO}_{2}$ es originado por procesos de descarbonatación de carbonatos marinos que se incorporan en el proceso de subducción que tiene lugar en la región, teniendo también importancia la contribución de origen magmático.

En la figura 6 se representan las concentraciones de $\mathrm{CO}_{2}$ y $\mathrm{O}_{2}$ en la atmósfera del suelo. Este diagrama binario nos permite obtener información sobre la existencia desde el punto de vista químico de diferentes reservorios geoquímicos que dan origen a las emanaciones difusas de $\mathrm{CO}_{2}$ en el Miravalles, como lo ha reflejado el análisis estadístico-gráfico del conjunto de los datos. En este diagrama se representan las principales líneas de mezcla entre los tres reservorios geoquímicos considerados para evaluar el origen de las emanaciones difusas de $\mathrm{CO}_{2}$ : (1) gas magmático $\left(\mathrm{CO}_{2}=100 \%\right.$ y $\left.\mathrm{O}_{2}=0 \%\right)$, (2) aire atmosférico $\left(\mathrm{CO}_{2}=0,03 \%\right.$ y $\left.\mathrm{O}_{2}=20,5 \%\right)$, y (3) gas biogénico. En este diagrama binario se representa también el gas fumarólico de Las 


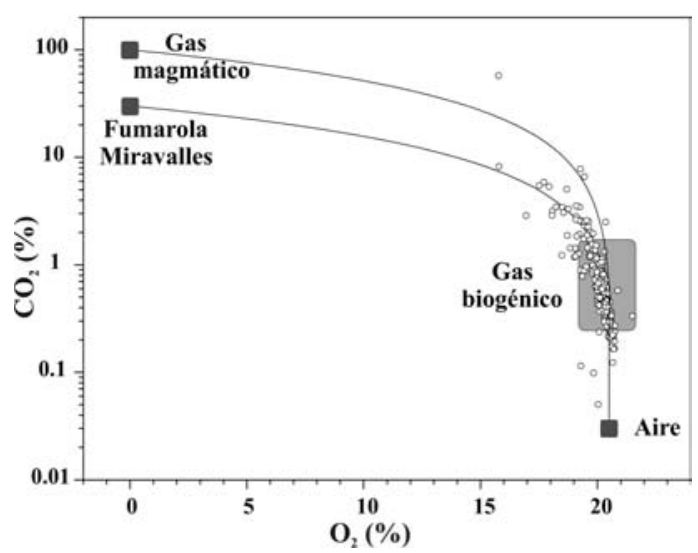

Fig. 6: Diagrama de correlación entre las concentraciones de $\mathrm{CO}_{2}(\%)$ y $\mathrm{O}_{2}(\%)$ en el gas del suelo del volcán Miravalles. La línea continua representa la línea de mezcla entre el gas volcánico y el reservorio del aire. En este diagrama se representa también el gas fumarólico de Las Hornillas.

Hornillas. Las dimensiones gráficas del reservorio del gas biogénico se seleccionaron considerando el rango de concentraciones de la población de fondo para el $\mathrm{CO}_{2}$ en la atmósfera del suelo en el Miravalles. La mayor parte de los datos de concentración de $\mathrm{CO}_{2}$ y $\mathrm{O}_{2}$ se ajustan bien a la línea de mezcla entre los reservorios geoquímicos aire atmosférico y gas biogénico con gases de origen profundo que ratifica lo que se ha estimado mediante el análisis estadístico: una perturbación del sistema volcánico-hidrotermal del Miravalles sobre las emanaciones difusas de $\mathrm{CO}_{2}$.

Con la finalidad de realizar una completa separación gráfica de los diferentes reservorios geoquímicos que se representaron en la Fig. 6, se construyó un diagrama binario adicional en el que se representa el contenido de $\mathrm{CO}_{2}$ (ppmV) en la atmósfera del suelo y sus correspondientes valores isotópicos de $\delta^{13} \mathrm{C}\left(\mathrm{CO}_{2}\right)$ (Fig. 7). En este nuevo diagrama se incluyen las líneas de mezcla entre los tres reservorios geoquímicos: (1) aire atmosférico $\left(\mathrm{CO}_{2}=355 \mathrm{ppmV}\right.$ y $\delta^{13} \mathrm{C}\left(\mathrm{CO}_{2}\right)=$ $-8 \%$ ), (2) gas magmático $\left(\mathrm{CO}_{2}=100 \% \mathrm{y}\right.$ $\delta^{13} \mathrm{C}\left(\mathrm{CO}_{2}\right)=-6 \%$ ) y (3) gas biogénico $(2640<$ $\mathrm{CO}_{2}<17600$ ppmV y $-25,7<\delta^{13} \mathrm{C}\left(\mathrm{CO}_{2}\right)<-$ $31,6 \%$ ) y la composición representativa del gas fumarólico de Las Hornillas $\left(\mathrm{CO}_{2}=30 \% \mathrm{y}\right.$ $\delta^{13} \mathrm{C}\left(\mathrm{CO}_{2}\right)=-1,8 \%$ o $)$. En este gráfico puede

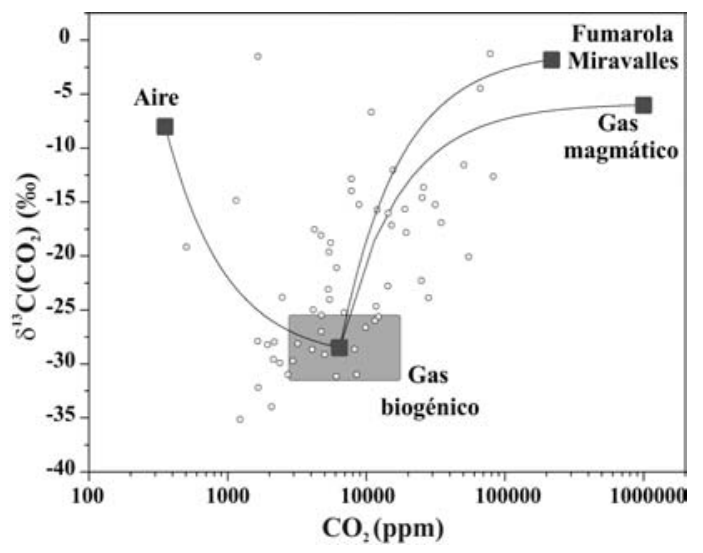

Fig. 7: Diagrama de correlación entre los valores de $\delta^{13} \mathrm{C}\left(\mathrm{CO}_{2}\right)$ y las concentraciones de $\mathrm{CO}_{2}(\mathrm{ppmV})$ en el gas del suelo. Las líneas continuas representan las líneas de mezcla entre el reservorio del aire y el gas biogénico, y entre el gas biogénico y el gas volcánico. En este diagrama se representa también el gas fumarólico de Las Hornillas.

observarse que la mayor parte de las muestras se localizan entre los reservorios volcánico y biogénico, lo que confirma el proceso de mezcla entre ambos y descarta una perturbación atmosférica para aquellas muestras que presentan una firma isotópica de $\mathrm{CO}_{2}$ relativamente más pesada. Los valores de $\delta^{13} \mathrm{C}\left(\mathrm{CO}_{2}\right)$ son utilizados para distinguir los diferentes orígenes del $\mathrm{CO}_{2}$ en las muestras de gas (Schwarcz, 1969; Hoefs, 1980), aunque estos no son determinantes para discernir el origen del $\mathrm{CO}_{2}$.

La estimación del $\mathrm{CO}_{2}$ total emitido en forma difusa a la atmósfera por el volcán Miravalles se realizó considerando el volumen positivo encerrado entre la superficie del área de estudio $\left(65 \mathrm{~km}^{2}\right)$ y el flujo de $\mathrm{CO}_{2}$ en 3-D, alcanzándose un valor de $12700 \mathrm{t} \mathrm{d}^{-1}$ de $\mathrm{CO}_{2}$. Si comparamos esta tasa de emisión con los valores obtenidos en distintos estudios de desgasificación difusa de $\mathrm{CO}_{2}$ en volcanes de América Central (Cuadro 2), podemos observar que es superior a la calculada para el volcán Irazú (Costa Rica), donde se estimó una emisión difusa total de $44 \mathrm{t}$ $\mathrm{d}^{-1}$ de $\mathrm{CO}_{2}$ para un área de $3,5 \mathrm{~km}^{2}$ (Galindo et $a l$., este volumen), y a la observada en el año 2000 para el volcán Poás, con una emisión difusa total de $\mathrm{CO}_{2}$ de $94 \mathrm{t} \mathrm{d}^{-1}$ en un área de $3,4 \mathrm{~km}^{2}$ (Melián et al., 2001). Por el contrario, si tenemos en cuenta la diferencia de las dimensiones del 
Cuadro 2

Emisión difusa total de $\mathrm{CO}_{2}$ para distintos volcanes de Centro América

\begin{tabular}{lcccr}
\hline Volcán & Año & $\begin{array}{c}\text { Área de estudio } \\
\left(\mathrm{km}^{2}\right)\end{array}$ & $\begin{array}{c}\Phi \mathrm{CO}_{2} \text { total } \\
\left(\mathrm{t} \mathrm{d}^{-1}\right)\end{array}$ & $\begin{array}{c}\Phi \mathrm{CO}_{2} / \mathrm{unidad}^{-1} \\
\mathrm{de} \mathrm{área}\left(\mathrm{g} \mathrm{m}^{-2} \mathrm{~d}^{-1}\right)\end{array}$ \\
\hline Irazú (CR) & $2001(1)$ & 3,5 & 44,2 & 13 \\
Poás (CR) & $2000(2)$ & 3,4 & 93,5 & 27 \\
Miravalles (CR) & 2000 & 65 & 12700 & 195 \\
Masaya (NI) & $1999(3)$ & 44 & 28800 & 655 \\
Cerro Negro (NI) & $1999(4)$ & 0,6 & 2800 & 4660 \\
San Salvador (SV) & $1999(5)$ & 266 & 4000 & 15 \\
Santa Ana (SV) & $2001(5)$ & 214 & 1400 & 7 \\
\hline
\end{tabular}

CR: Costa Rica; NI: Nicaragua; SV: El Salvador

(1) Galindo et al., en este volumen; (2) Melián et al. (2001); (3) Pérez et al. (2000); (4) Salazar et al. (2001) y (5) Salazar et al. (2002).

área de estudio, la emisión difusa total de $\mathrm{CO}_{2}$ en el volcán Miravalles es muy inferior a la observada para el volcán Masaya (Nicaragua) donde se estimó una emisión difusa de $\mathrm{CO}_{2}$ total de $28800 \mathrm{t} \mathrm{d}^{-1}$ en un área de $44 \mathrm{~km}^{2}$ (Pérez et al., 2000) y a la encontrada en el volcán Cerro Negro (Nicaragua) donde se estimó una emisión difusa total de $2800 \mathrm{t} \mathrm{d}^{-1}$ para un área de $0,6 \mathrm{~km}^{2}$ en el año 1999 (Salazar et al., 2001). Estas diferencias en la tasa de emisión pudieran tener relación con las características volcano-tectónicas del arco volcánico centroamericano.

\section{CONCLUSIONES}

La distribución espacial del flujo difuso de $\mathrm{CO}_{2}$ muestra que las principales anomalías se encuentran localizadas en los sectores SE y SO del volcán Miravalles. Estas anomalías no presentan una buena correlación espacial con los niveles de concentración de vapor de $\mathrm{Hg}^{0}$ medidos en al ambiente superficial del volcán Miravalles, aunque se puede observar concordancia entre la emisión difusa de $\mathrm{CO}_{2}$ y los niveles de vapor de $\mathrm{Hg}^{0}$ en la parte $\mathrm{SO}$ del volcán coincidiendo con los ejes volcano-estructurales. El volcán Miravalles emite a la atmósfera $12700 \mathrm{t} \mathrm{d}^{-1}$ de $\mathrm{CO}_{2}$ estimados de forma conservativa en un área de $65 \mathrm{~km}^{2}$, tasa de emisión superior a la observada en otros volcanes de América Central. Las características químicas e isotópicas del
$\mathrm{CO}_{2}$ en la atmósfera del suelo, indican una mezcla de fuentes profundas y superficiales y donde la contribución magmática a las emisiones difusas de $\mathrm{CO}_{2}$ en el volcán Miravalles es considerable. El monitoreo de estas emisiones difusas puede ser muy útil para la vigilancia volcánica del Miravalles, con el aporte de un enfoque multidisciplinar.

\section{AGRADECIMIENTOS}

Este trabajo no hubiera podido ser realizado sin la colaboración y ayuda de nuestro amigo Juan Carlos Mesa, quien de forma desinteresada nos ayudó a la realización de la campaña de campo. Queremos agradecer al Proyecto Geotérmico Miravalles (PGM) que nos brindó tanto el apoyo logístico durante la realización de la campaña, como el hospedaje en sus instalaciones, así como a todo el personal del laboratorio de Química y Geología, a los conductores y cocineros del complejo. También queremos agradecer muy especialmente a nuestros ayudantes de campo Francisco, Luis, Mainor, y Orontes, ya que sin ellos no habríamos podido llevar a cabo este trabajo, y muy especialmente a Fernando Molina, Eduardo Vega, Oscar Rodríguez y Oscar Murillo quienes hicieron más agradable nuestra estancia en las instalaciones del PGM. Agradecemos también al Laboratorio de Química de Terremotos de la Universidad de Tokio, que nos ha permitido realizar 
los análisis isotópicos de las muestras en sus instalaciones. Este trabajo se realizó gracias a la fundación ITER y CajaCanarias (Tenerife, España).

\section{REFERENCIAS}

ALVARADO, G. E., 2000: Los volcanes de Costa Rica: Geología, historia y riqueza natural. - [2 $2^{\mathrm{a}}$ ed.] 269 págs. EUNED, San José, Costa Rica.

BAUBRON, J. - C., ALlARD, P. \& TOUTAIN, J., 1990: Diffuse volcanic emissions of carbon dioxide from Vulcano Island, Italy. - Nature, 344: 51-53.

CHIODINI, G., FRONDINI, F. \& RACO, B., 1996: Diffuse emission of $\mathrm{CO}_{2}$ from the Fossa Crater, Vulcano Island (Italy). - Bull. Vulcanol. 58: 41-50.

CRESSIE, N.A.C., 1990: The origins of Krigging. - Math. Geol. 22: 239 .

GALINDO, I., MELIÁN, G., SALAZAR, J., HERNÁNDEZ, P., PÉREZ, N., RAMÍREZ, C., FERNÁNDEZ, M., ALVARADO, G., SUMINO, H. \& NOTSU, K., 2004: Emisión difusa de dióxido de carbono en el volcán Irazú, Costa Rica. - Rev. Geol. Amér. Central, 30 (este volumen).

GERLACH, T. \& GRAEBER, E., 1985: Volatile budget of Kilauea volcano. - Nature, 313: 273-277.

GIGGENBACH, W., 1987: Redox process governing the chemistry of fumarolic discharges from White Island, New Zeland. - Appl. Geochem. 2: 143161.

GIGGENBACH, W., 1996: Chemical composition of volcanic gases. - Inst. Geol. Nuclear Sci. 31: 221-256.

HERNÁNDEZ, P., SALAZAR, J., SHIMOIKE, Y., MORI, T., NOTSU, K., SATO, M. \& PÉREZ, N., 2001a: Diffuse emission of $\mathrm{CO}_{2}$ from Miyakejima volcano, Japan. - Chem. Geol. 177: 175-185.

HERNÁNDEZ, P., NOTSU, K., SALAZAR, J., MORI, T., NATALE, G., OKADA, H., VIRGILI, G., SHIMOIKE, Y., SATO, M. \& PÉREZ, N., 2001b: Carbon dioxide degassing by advective flow from Usu volcano, Japan. - Science, 292: 83-86.

HINKLE, M. \& KILBURN, J., 1979: The use of vacutainer tube for collection of soil samples for helium analysis. - U. S. Geol. Surv. Open File Rep., 79: 1441.

HIRABAYASHI, J., OSSAKA, J. \& OZAWA, T., 1982: Relationship between volcanic activity and chemical composition of volcanic gases. A case study on the Sakurajima Volcano. - Geochem. J. 16: 11-21.

HOEFS, J., 1980: Stable isotopes geochemistry. - 208 págs. Springer, Berlín.

JOURNEL, A., 1988: Principles of environment sampling. En: KEITH, C. (ed.), Amer. Chem. Soc.: 45-72.

LESCINSKY, D., CONNOR, C. \& STOIBER, R., 1987: Soil mercury study of thermal areas, Rincón de la Vieja Volcano, Costa Rica. - Geothermics, 16(2): 159-168.

MARTINI, M., GIANNINI, L. \& CAPACCIONI, B., 1991: Geochemical and seismic precursors of volcanic activity. - Acta Vulcanol. 1: 7-11.

MELIÁN, G., GALINDO, I., SALAZAR, J., HERNÁNDEZ, P., PÉREZ, N., RAMÍREZ, C., FERNÁNDEZ M. \& NOTSU, K., 2001: Spatial and secular variations of diffuse $\mathrm{CO}_{2}$ degassing from Poás volcano, Costa Rica, Central America. - [res.] AGU Fall Meeting, San Francisco. EOS, Trans., AGU, 82: F1332.

PÉREZ, N., WAKITA, H., LOLOK, D., PATIA, H., TALAI, B. \& MCKEE, C., 1996: Anomalous soil gas $\mathrm{CO}_{2}$ concentrations and relation to seismic activity at Rabaul caldera, Papua New Guinea. - Geogaceta, 20: 1000-1003.

PÉREZ, N., MELIÁN, G., SALAZAR, J., SABALlOS, A., ÁLVAREZ, J., SEGURA, S., HERNÁNDEZ, P. \& NOTSU, K., 2000: Diffuse degassing of $\mathrm{CO}_{2}$ from Masaya caldera, Nicaragua, Central América. - [res.] AGU Fall Meeting, San Francisco. EOS, Trans., AGU, 81, 48: F1318.

ROGIE, J., KERRICK, D., SOREY, M., CHIODINI, G. \& GALLOWAY, D., 2001: Dynamics of carbon dioxide emission at Mammoth Mountain, California. Earth Planet. Sci. Lett. 188: 535-541.

SALAZAR, J., HERNÁNDEZ, P., PÉREZ, N., MELIÁN, G., ÁLVAREZ J., SEGURA, F. \& NOTSU, K., 2001: Diffuse Emission of Carbon Dioxide from Cerro Negro Volcano, Nicaragua. - Geophys. Res. Lett. 28: 4275-4278.

SALAZAR, J.M.L., PÉREZ, N.M., HERNÁNDEZ, P.A., SORIANO, T., BARAHONA, F., OLMOS, R., CARTAGENA, R., LÓPEZ, D.L., LIMA, R.N., MELIÁN, G., GALINDO, I., PADRÓN, E., SUMINO, H., \& NOTSU, K., 2002: Precursory diffuse carbon dioxide degassing signature related to a 5.1 magnitude earthquake in El Salvador, Central América. Earth Planet. Sci. Lett. 205: 81-89.

SANO, Y., URABE, A., WAKITA, H., CHIBA, H. \& SAKAI, H., 1985: Chemical and isotopic composition 
of gases in geothermal fluid in Iceland. - Geochem. J. 19: 135-148.

SANO, Y. \& MARTY, B., 1995: Origin of carbon in fumarolic gas from island arcs. - Chem. Geol. 119: 265-274.

SCHWARCZ, H., 1969: The stable isotopes of carbon. - En: WEDEPOHL, K.H. (ed.), Handbook of Geochemistry. Springer, Berlín.
SINCLAIR, A., 1974: Selection of thresholds in geochemical data using probability graphs. - J. Geochem. Explor. 3: $129-149$.

SNYDER, G., POREDA, R., HUNT, A. \& FEHN, U., 2001: Regional variation in volatile composition: isotopic evidence for carbonate recycling in the Central American volcanic arc. - Geochem. Geophys. Geosystems, 2, paper $2001 \mathrm{GC} 000163$. 